\title{
Continuing Bonds Expressions After Loss: Role of Bereavement Type and Impact Of Event
}

\section{Introduction}

Continuing bonds (CB) are defined as efforts to continue an emotional connection to the deceased (Field, Nichols, Holen \& Horowitz, 1999) with the aim of sustain connectedness and bond rather than ending the relationship with the deceased (Field, 2008). The CB expressions may include looking at photos, reminiscing, engaging in memorials and rituals, talking to the deceased, dreams, and holding onto special possessions. Although some research report that maintaining an ongoing connection with their deceased loved one is comforting and meaningful (see Klass, Silverman \& Nickman, 1996), there are other research findings reporting that $C B$ is associated with more severe bereavementrelated symptomatology (Stroebe \& Schut, 2005).

In this respect, the aim of this study is to explore $C B$ expressions considering the impact of the event and complexity of bereavement.

\section{Method}

Participants: The sample consists of 423 bereaved people (77\% females) with loss of a significant person in their life (e.g. mother, father, spouse, brother, child, partner, close relative, or a loved friend, and so on).

Materials: The participants completed the survey consisted of demographic information form and Turkish versions of;

- Traumatic Grief Inventory-Self Report Version (TGI) (Boelen et al., 2018),

Impact of Event Scale-Revised (IES) (Weiss \& Marmar, 1997)

Continuing Bond Interview (CBI) (Field, Packman \& Carmack, 2007).
Results

Participants reported a high frequency of $\mathrm{CB}$ utilization in the past month, with the most commonly endorsed reminiscing the deceased with others $(83,5 \%)$, focusing fond memories $(83,5 \%)$ and using belongings to feel closer $(47.3 \%)$ to the deceased (see Table 1). When asked whether the use of a specific bond made them feel "better", "worse", or "both", the majority of participant reported feeling increased comfort when identificate with the qualities of the deceased $(85,3 \%)$. While having intrusions about the presence of the deceased makes the participants feel "worse" (55\%), using belongings is described as making participants feel "both" better/worse (55\%) (Table 1).

Table 1:Endorsement of CB Expressions

\begin{tabular}{|c|c|c|c|c|}
\hline Continuing Bond (CB) & $\begin{array}{c}\text { Endorsed } \\
\text { CB }\end{array}$ & Better & Worse & Both \\
\hline
\end{tabular} \begin{tabular}{|l|c|c|c|c|}
\hline Expression & $\mathrm{N}(\%)$ & $(\%)$ & $(\%)$ & $(\%)$ \\
\cline { 2 - 6 }
\end{tabular} \begin{tabular}{|l|c|c|c|c|}
\hline Continued Connection & $118(27,9)$ & 40,68 & 12,71 & 46,61 \\
\hline
\end{tabular} \begin{tabular}{|l|l|l|l|l|l|}
\hline Belongings & $201(47,3)$ & 36,32 & 8,96 & 54,73 \\
\hline
\end{tabular} \begin{tabular}{|l|l|l|l|l|l|}
\hline Associated Places & $183(43,1)$ & 46,45 & 13,11 & 40,44 \\
\hline
\end{tabular} \begin{tabular}{|l|l|l|l|l|l|}
\hline Fond Memories & $355(83,5)$ & 49,58 & 5,07 & 45,35 \\
\hline
\end{tabular} \begin{tabular}{|l|c|c|c|c|}
\hline Dreams & $183(43,1)$ & 60,11 & 31,15 & 8,74 \\
\hline
\end{tabular} \begin{tabular}{|l|l|l|l|l|l|}
\hline Everyday Choices & $96(22,6)$ & 67,71 & 6,25 & 26,04 \\
\hline
\end{tabular} \begin{tabular}{|l|c|c|c|c|}
\hline Reunited & $130(30,6)$ & 75,38 & 4,62 & 20,00 \\
\hline
\end{tabular} \begin{tabular}{|l|l|l|l|l|}
\hline Identification & $184(43,3)$ & 85,33 & 1,63 & 13,04 \\
\hline
\end{tabular} \begin{tabular}{|l|l|l|l|l|l|}
\hline Wishes of Deceased & $149(35,1)$ & 78,52 & 4,03 & 17,45 \\
\hline
\end{tabular} \begin{tabular}{|l|l|l|l|l|l|}
\hline $\begin{array}{l}\text { Reminisced with } \\
\text { others }\end{array}$ & $355(83,5)$ & 37,75 & 13,80 & 48,45 \\
\hline
\end{tabular} \begin{tabular}{|l|l|l|l|l|}
\hline Contact with the spirit & $123(28,9)$ & 73,98 & 4,07 & 21,95 \\
\hline
\end{tabular} \begin{tabular}{|l|c|c|c|c|}
\hline Intrusion & $100(23,5)$ & 13,00 & 55,00 & 32,00 \\
\hline
\end{tabular} Notably, binary logistic regressions showed that severity of grief as measured by the TGI is predictive of the use of all types of CB except having dreams and intrusions.
Specifically, higher level of grief is related to having a continuing connection with the deceased $(E=1.04, W=13.12, p=.00)$, using

belongings to feel comfort $(E=1.05, W=24.77$ $p=.00$ ), feeling drawn to places associated with the deceased $(E=1.03, W=11.86, p=.00)$ focusing on fond memories $(E=1.05, W=9.79$ $p=.00$ ), making everyday decisions based on preferences of the deceased $(E=1.04, W=$ $12.60, p=.000$ ), having thoughts about being reunited in the future $(E=1.04, W=5.00, p=$ .02 ), identification with the qualities of the deceased $(E=1.04, W=14.01, p=.00)$, trying to live up to wishes of the deceased $(E=1.05$ $, W=19.72, p=.00)$, reminiscing with others about the deceased $(E=1.03, W=5.60, p=.01)$ attempting to contact with the deceased's spirit $(E=1.03, W=8.08, p=.00)$, conducting commemorative activities $(E=1.03, W=6.82$ $\mathrm{p}=.00)$.

Although, there is no significant relationship with the CB endorsement and impact of event regression analysis showed that unexpectedness of loss is predictive of severity of the grief $(E=.96, W=14.16, p=.00)$.

Figure 1: Commemorative Activities - Having Memorial Tattoo

- Doing Charity Work

Dedicating Something to Planting Memorial Trees orial Trees - Naming Someone After Having a Memorial Object

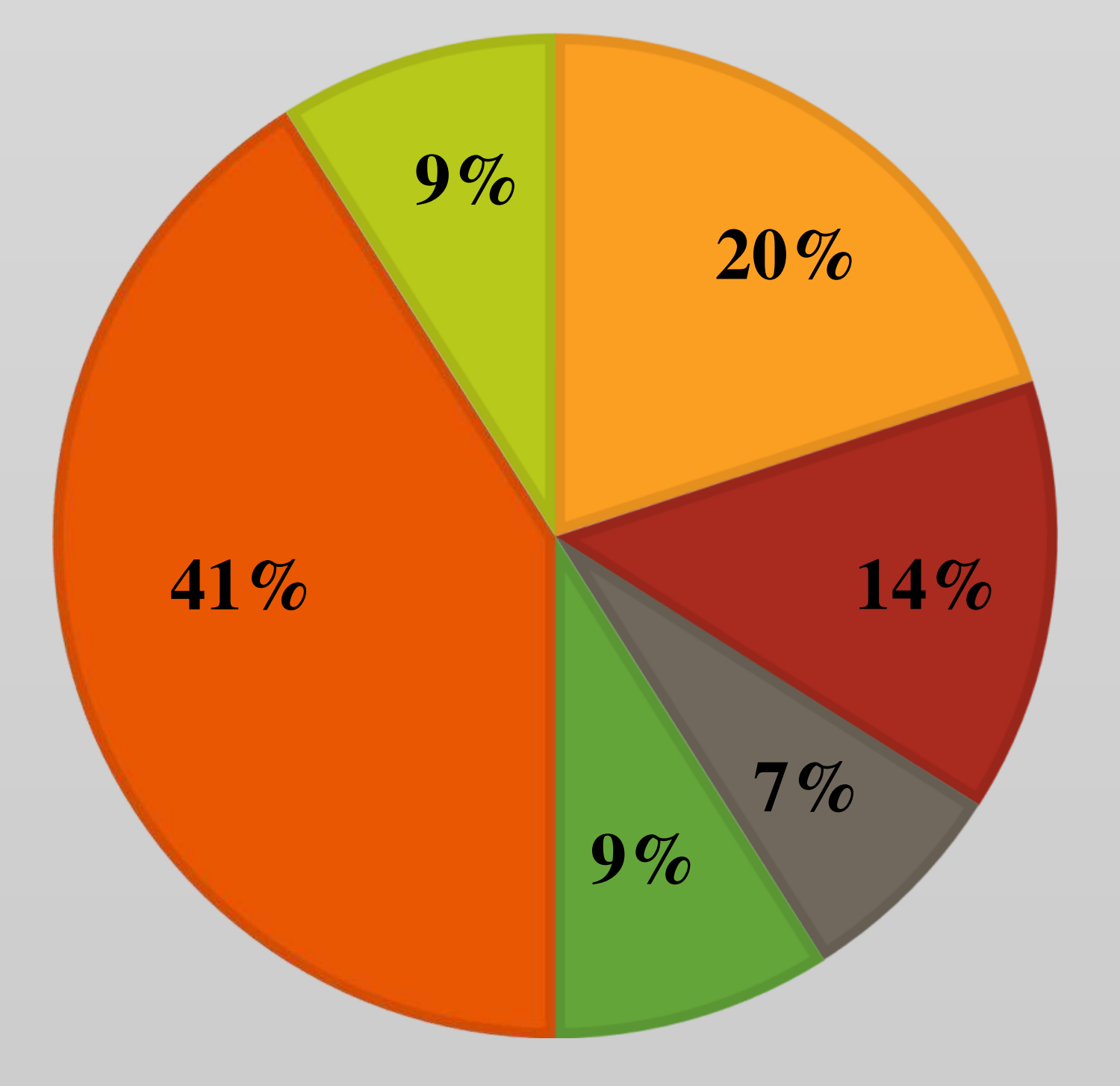

In terms of remembrance activities, $53(\% 12,5)$ participants reported that they carried out a permanent activity. Specifically, results showed that most common activity is naming children after someone who passed away while getting a memorial tattoo is placed as second in ranking (Figure 1). The average score of this group on the $\mathrm{TGI}$ is $57,7(\mathrm{SD}=18,7)$ as scores of 30 and higher indicating the presence of clinically significant grief

\section{Conclusion}

The current study has a contribution to the bereavement literature as it includes qualitative and quantitative data. Although the majority of CB were viewed as comforting following the loss participants having severe grief symptoms more likely to report $C B$ expressions without regard to impact of event. As such, we noted an inconsistency in the literature regarding the adaptiveness of relinguishing the bonds to the deceased person. Turkish religious rituals and customs regarding the grief may be an explanation of this outcome that mainly does not promote continuing emotional connection to the deceased. In this respect, it is important to take consideration of the social and communal nature of continuing bonds as Klass (2006) proposed. Within this framework, naming the children after a family member who has passed away is a very common commemorative activity in Turkish culture as results indicated.

\section{References}

Boelen P. A., Manik J. Djelantik A. A. A., Keijser Jos de. Lenferink, L. I. M \&. Smid G. E. (2018) Further validation of th persistent complex bereavelf Report (TGI-SR). A ment disorder and prolonged grief disorder, Death Studies, 43:6, 351-364

Field, N.,Nichols C., Holen, A\& Horowitz, M. (1999). The relation bereavement. Journal of consulting and clinical psychology. 67.

Field, N. P.,Packman, W., \& Carmack, B. J. (2007). Continuing bonds interview. Unpublished data.

Klass, D., Silverman, P., \& Nickman, S. L. (Eds.) (1996) Continuing bonds: New understandings of grief. Washington DC: Taylor \& Francis.

Stroebe, M. S., \& Schut, H. (2005). To continue or relinquish bonds: $A$ review of consequences for the bereaved. Death Studies, 29, 477-494

Weiss, D. S., \& Marmar, C. R. (1997). The Impact of Event Scale-Revised. In J. P. Wilson \& T. M. Keane (Eds.), Assessing psychological trau
US: Guilford Press. 